\title{
Conrad Geßner als Bibliograph und Enzyklopädist
}

\author{
Der Zusammenbruch der mittelalterlichen artes liberales
}

\section{Von Josef MaYerhöfer}

Conrad Gessner gilt heute allgemein als einer der ersten großen Naturforscher der Neuzeit. Das zunehmende Interesse für die Geschichte der Naturwissenschaften hat in der letzten Zeit dazu beigetragen, den Ruhm Geßners in dieser Hinsicht nur noch zu verstärken. Man sollte dabei aber eine andere Seite in Geßners Wesen nicht vergessen: daß er eigentlich von der Philologie und Lexikographie herkam und als Verfasser der Bibliotheca universalis auch in der Geschichte des Buchwesens einen hervorragenden Platz einnimmt ${ }^{1}$. In einer Zeit, in der es noch nicht die so verhängnisvolle Trennung von Natur- und Geisteswissenschaften gab, wandte Geßner seinen ihm angeborenen Sammel- und Ordnungssinn zunächst auf die Sprachen an und schrieb Lexika, er sammelte Buchtitel und schrieb eine Bibliographie, dann sammelte er literarische Belegstellen und schrieb Tierbücher, und erst gegen Ende seines kurzen Lebens ging er in die Natur selbst hinaus und sammelte, ordnete und beschrieb, was er fand. Keine dieser Arbeiten faßte er oberflächlich auf, sondern suchte stets der Sache auf den Grund zu gehen und das Beste zu geben, wozu er imstande war. Geßners bibliographische Tätigkeit war also nicht die schrullige Nebenbeschäftigung eines geborenen Naturforschers, sondern Ausfluß eines Charakterzuges, der nicht nur bei $\mathrm{ihm}$, sondern auch bei anderen Persönlichkeiten in der abendländischen Geistesgeschichte anzutreffen ist.

Das meisterhafte Produkt von Geßners bibliographischer Tätigkeit ist ein 1545 bei Froschauer in Zürich erschienener Foliant von etwa 650 Seiten, die Bibliotheca universalis (Abb. 1). Es handelt sich um ein alphabetisches Nachschlagewerk, bei dem unter dem Namen der Autoren ihre Werke verzeichnet sind. Wer es aufschlägt, ist zunächst überrascht, denn die Anordnung ist wohl alphabetisch, aber nach dem Vornamen, nicht nach Familien- oder Beinamen. Cicero findet sich also unter Marcus Tullius Cicero,

1 Vgl. z.B. Allgemeine Enzyklopädie der Wissenschaften und Künste, herausgegeben von Johann Samuel Ersch und Johann Gottfried Gruber, Band I 64, Leipzig 1857, S. 345-358. - J. Chr. BAY, Conrad Gesner, the Father of Bibliography, in Papers of the Bibliographical Society of America 10 (1916) 53-88. - Georg SchneIDER, Handbuch der Bibliographie, 4. Auflage, Leipzig 1930, S. 48. 


\section{BIBLIOTHECA}

\section{Vniuerfalis, fiue Catalogus omnis}

umfcriptorum locupletiffimus, in tribus linguis, Lacina, Græca, $8 \mathrm{Hes}$ braica: extantium \& non extantiú, ueterum \& recentiorum in huncufक diem, doctorum \& indoctorum, publicatorum \& in Bibliothecis laten= tium. Opus nouum, \& nő Bibliothecis tantum publicis priuarisue ins ftituendis neceffarium, fed ftudiofis omnibus cuiufcun $\phi$ artis aut fcientix ad ftudia melius formanda utilifimum : authore CONRADO GESNERO Tigurino doctore medico.

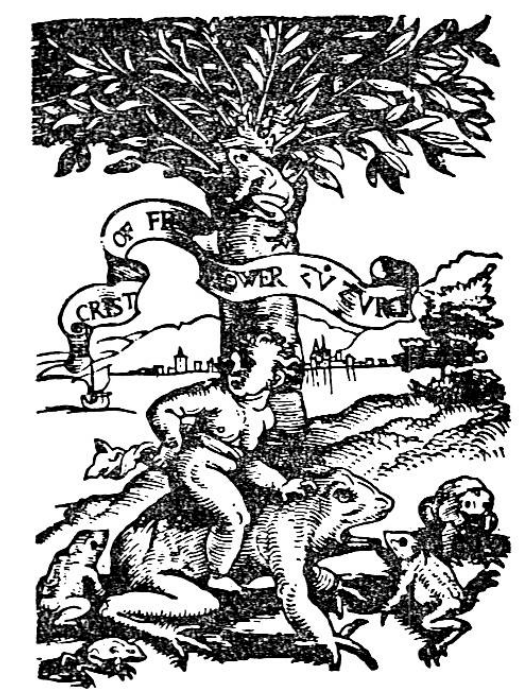

TIGVRI APVD CHRISTOPHOR VM Frofchouenum Mernfe Septembri, Anno $M, D, X L V$.

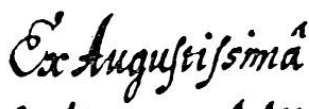

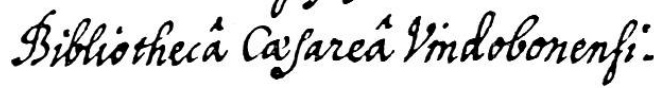

Abb. 1. Titelblatt der Bibliotheca universalis von 1545 
Geßner unter Conradus Gesnerus. Letzteres entspricht noch dem mittelalterlichen Brauch, gemäß dem der Taufname der Hauptname war und nur gelegentlich Beinamen, aber kein Familienname existierte. Mittelalterliche Autoren werden auch in modernen Bibliothekskatalogen unter dem Vornamen eingereiht. Zu Geßners Zeiten hatte sich allerdings schon der Familienname durchgesetzt. Obwohl die Vornamen, wie Geßner selbst zugibt, oft weniger bekannt sind, entschied er sich doch für die Einreihung unter diesen, weil er zur Vermeidung von Verwechslungen die Vornamen unbedingt brauchte ${ }^{2}$, eine Einreihung der «Vor»-Namen hinter dem Familiennamen (wie jetzt allgemein üblich) nicht in Erwägung zog. Für jene Leser, die den Vornamen eines Autors nicht wissen, ist ein langes Verzeichnis der Autoren mit den Vornamen beigegeben.

Geßners Ziel war, die antiken Autoren möglichst vollständig, von den modernen wenigstens die wichtigeren zu erfassen, und zwar ohne Rücksicht auf Beruf, Religion oder Lehrmeinung - ein in einer Zeit beginnender Glaubenskämpfe (bei denen auch sein Vater gefallen war) besonders bemerkenswerter Gesichtspunkt ${ }^{3}$. Es sind nur die in Latein, Griechisch oder Hebräisch verfaßten Bücher erwähnt. Daß Geßner die Bücher in den Volkssprachen weglassen konnte, beweist deren geringe Bedeutung in einer Zeit, in der die Wissenschaft noch durchaus international war. Die einzelnen Artikel sind von sehr verschiedener Länge, je nach der Bedeutung des Autors. Sie bringen zuerst kurze biographische Notizen, oft mit eingestreuten persönlichen Bemerkungen. Dann sind die gedruckt erschienenen Bücher verzeichnet, vielfach mit Kapitelüberschriften und Inhaltsangaben einzelner Abschnitte. Dazu kommen Verlagsort und Verlag, oft auch das Erscheinungsjahr. Manchmal sind auch noch ungedruckte und sogar nur projektierte Werke erwähnt, insgesamt sind etwa 12000 Buchtitel verzeichnet.

Nach heutiger Terminologie handelt es sich bei der Bibliotheca universalis um eine nach Personen geordnete internationale Allgemeinbibliographie, und zwar um die erste ihrer Art, weshalb sie aus der Geschichte des Buchwesens nicht wegzudenken ist. Sie wurde noch zweimal neu bearbeitet und gedruckt, hat aber keine eigentliche Nachfolge gefunden, was sich aus der immer schnelleren Zunahme der Buchproduktion erklärt. Geßner kannte den Begriff Bibliographie im heutigen Sinn noch nicht. Er faßte sein Werk als eine ideale Bibliothek und als Handbuch für die Einrichtung neuer pri-

2 Bibliotheca universalis, Epistola nuncupatoria, S. (6).

3 Ebenda, Ad lectores (Rückseite des Titelblattes). 


\section{PANDECTARVMSIVE Partitionum uniuerfalium Con radi Gefneri Tigurini, medici \& philofophixprofeffo ris, libri $\overline{\mathbf{X x I}_{\text {}}}$}

ADIECTORES.

S B C V N D V S hiC B I B L I O T H B C A B nofire Tomus ef, torius philofophice 2 omnium bonarum artium atø ftydiorum Locos communes $\&$ Ordines uniuerfales fimul \& particulares compleferen. Vos lacrum hocingens ad fua quifos ftudia reportate, 8 grati cftote authori propter tantum Opus feliciter diuina gratia inchoatum : non quod idem abfoluendum unquam ab ipfo expeetari debeas, fed ut quifog fibi pro ftudiorum fuo rum ufu id accommodec. Licebit enim fudiofis quicquid ufquam memoratu dignum les gendo occurtet, facile huc in fuos quafi nidos recondere, unde fuo tempore rurfus deprop mant. Et procliue quidē hoc erit factu illi, qui philolophiz partes atç冖 differentias cognouerit,aut faltem Titulos, quos fingulis in hoc Volumine libris Indicis uice fusturos preffiximus, iterum atq̧ iterum infpexerito

Librorum enumeratio fequente pagina continetur:

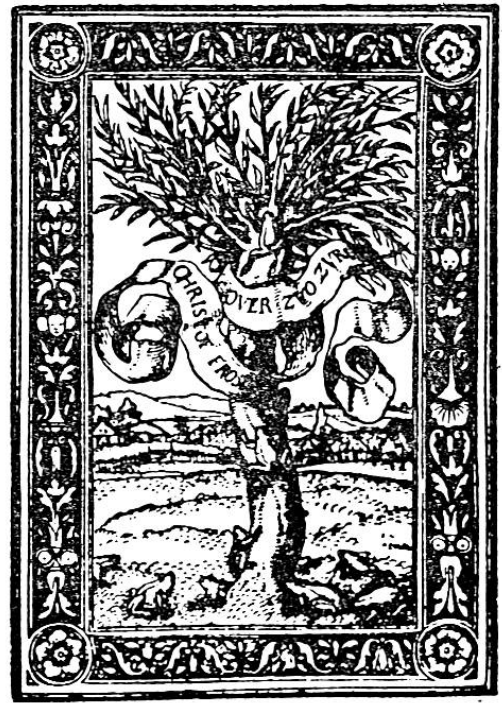

Extugugtifsinâ Bifliotherâ Cayareâ Vitidobonenf:

TIGVRI EXCVDEBAT CHRISTOPHORVS

Frofchourerus, Anno M. D. XL V III.

Abb. 2. Titelblatt der «Pandekten», 1548 
vater und öffentlicher Bibliotheken auf. In der Vorrede wird die Wichtigkeit von Bibliotheken besonders betont ${ }^{4}$.

Fast unerfindlich ist, wie Geßner im Alter von 29 Jahren schon ein so reiches bibliographisches Material zusammengetragen hatte. In der Vorrede erwähnt er seinen unermüdlichen Fleiß im Anlegen von Notizen und gibt auch an, daß er die verfügbaren Kataloge der Büchermessen, ferner die Kataloge der Vatikanischen und anderer Bibliotheken benützt hat, schließlich auch die Hinweise bei antiken und mittelalterlichen Autoren, wie Suidas usw. ${ }^{5}$ Man erkennt aber sofort, daß die «Bibliotheca» nicht eine bloße Kompilation ist, sondern daß Geßner die erwähnten Bücher jedenfalls genügend gekannt hat, um ein Urteil darüber abgeben zu können.

Die Arbeit an der Bibliotheca universalis veranlaßte aber Geßner, alsbald einen weiteren Schritt zu tun, nämlich den von einer bloßen Bibliographie nach dem Alphabet der Verfasser zu einer enzyklopädischen Ordnung. Er fühlte das Bedürfnis, die gewaltige Menge von Büchern auch nach der sachlichen Seite hin zu erschließen. Es ist dies ein echtes bibliothekarisches Anliegen. Wer heute eine öffentliche Bibliothek besucht, erwartet dort mindestens einen Autorenkatalog und einen Sachkatalog vorzufinden, wobei es von letzterem bekanntlich zwei Typen gibt: den systematischen Katalog und den (alphabetisch geordneten) Schlagwortkatalog. Diese ganze Sachlage war Geßner vollkommen geläufig, obwohl er kein Bibliothekar war und die Bibliothekare zu seiner Zeit kaum so präzise Anforderungen an die Bibliotheksverwaltung gestellt haben. Schon auf der Rückseite des Titelblattes seiner «Bibliotheca» verspricht Geßner einen zweiten und dritten Band. Sie sollten das gleiche Titelmaterial nochmals bringen zuzüglich der im Autorenalphabet nicht unterzubringenden anonymen Werke, aber das einemal systematisch, das anderemal nach dem Alphabet der Sachbegriffe geordnet. Dieses Unternehmen war freilich nicht so leicht in die Tat umzusetzen. 1548 erschien: Pandectarum sive partitionum universalium Conradi Gesneri libri XXI, ebenfalls bei Froschader, als Folioband von etwa 400 Seiten (Abb. 2). Im Vorwort klagt der Verfasser, daß er sofort nach der Veröffentlichung der «Bibliotheca » von Freunden und vom Verlag gedrängt worden sei, sein Versprechen einzulösen und den 2. und 3. Band fertigzustellen; diesem Verlangen komme er nun seufzend nach. Die «Pandekten» sind aber nur der systematische Teil, also der 2.Band. Von dem versproche-

4 Ebenda, Epistola nuncupatoria, S. (2).

5 Ebenda, S. (3). 
nen 3. Band, also dem alphabetischen Sachverzeichnis, war Geßner abgekommen, denn, so sagt er, wer sich ernstlich für einen bestimmten Gegenstand interessiert, wird auch wissen, unter welcher systematischen Gruppe er ihn zu suchen hat ${ }^{6}$.

Geßner hatte sich vorgenommen, das ganze Material in 21 Wissensgebiete aufzuteilen; entgegen der Ankündigung auf dem Titelblatt fehlen aber die beiden Gruppen Medizin und Theologie. Die Abteilung Theologie (Gruppe 21) erschien ein Jahr später (1549) separat als Partitiones theologicae pandectarum universalium, während der medizinische Teil (Gruppe 20) überhaupt nicht veröffentlicht wurde, weil Geßner stets ein noch besseres System anstrebte, aber damit überhaupt nicht fertig wurde.

Ungeachtet dieser Unvollkommenheiten liegt den «Pandekten» Geßners ein echtes System der Wissenschaften zugrunde, das einen ausgezeichneten Einblick in das Geistesleben seiner Zeit bietet. Wir wollen darauf näher eingehen. Wenn man sich fragt, wie ein Gelehrter überhaupt dazu kommt, ein solches System aufzustellen, so zeigen sich folgende Möglichkeiten: 1. der philosophische Gesichtspunkt: was kann der Mensch wissen? 2. der pädagogische Gesichtspunkt: wie soll der Unterricht gegliedert werden ? 3. der bibliographische Gesichtspunkt: wie sollen Bücher (bzw. Buchtitel) am besten geordnet werden ? In der abendländischen Geistesgeschichte erscheinen diese drei Gesichtspunkte manchmal streng getrennt, manchmal eng miteinander verbunden. Geßner hat sich, soviel bekannt, nicht von philosophischen oder pädagogischen Überlegungen leiten lassen, sondern ist als praktischer Bibliograph an die Sache herangegangen. Er hat keinen Ehrgeiz darin gesehen, ein möglichst neuartiges System der Wissenschaften aufzustellen, sondern bediente sich vielmehr im wesentlichen herkömmlicher Kategorien, die er aber dem Stand der Wissenschaften seiner Zeit anpaßte. Gerade darum ist aber sein System als Kulturdokument so interessant. Um es voll zu würdigen, müssen wir etwas ausholen ${ }^{7}$.

Die Frage nach einem System der Wissenschaften wurde in der klassischen Antike kaum gestellt, wenigstens nicht in unserem Sinne, denn einerseits waren die Wissenschaften noch nicht so abgegrenzt wie heute, anderseits auch nicht so spezialisiert. Gelegentlich finden sich jedoch Einteilun-

6 Pandekten, Praefatio, S. (6).

7 Vgl. z. B. Erich Podach, Der Begriff der Wissenschaft und die versuchten Wissenschaftseinteilungen, Berlin 1927 (Diss.). - Kurt KaAtz, Das Ordnungsgefüge der Wissenschaften. Zur Geschichte der Wissenschaftseinteilungen, Berlin 1949 (Diss.). - W.C.BERwICK SAYERS, An Introduction to Library Classification, 9 th edition, London 1955. 
gen, z.B. bei Aristoteles ${ }^{8}$, der zwischen theoretischer Philosophie (Physik, Metaphysik, Mathematik), praktischer Philosophie (Ökonomie, Politik) und "poietischer» Philosophie (Kunst, Technik) unterscheidet. PLATON teilt etwa ein in erkennende und ausübende Wissenschaft ${ }^{9}$; eine andere Einteilung in Logik, Physik und Ethik, die den seelischen Grundfunktionen Vernunft, Sinneswahrnehmung und Wollen entspricht, wird ihm zugeschrieben ${ }^{10}$. In der Politeia nähert sich Platon aber den Pythagoreern, deren Ansichten er besonders durch den mit ihm befreundeten ARchytas von Tarent kennengelernt hatte. Archytas lehrte, daß man einen Einblick in die Natur am besten von den mathematischen Disziplinen her gewinnen könne; dazu gehören: Astronomie, Geometrie, Arithmetik, Sphärik, besonders aber Musik ${ }^{11}$. Platon fügte dieser Aufzählung noch die Harmonik und die Dialektik hinzu ${ }^{12}$.

Diese rein philosophischen Einteilungen der Wissenschaften erlangten aber niemals eine solche Bedeutung wie jene pädagogische Einteilung in die sieben artes liberales ${ }^{13}$, unter denen allerdings die obenerwähnten mathematischen Disziplinen im Sinne von Archytas und Platon eine beson-

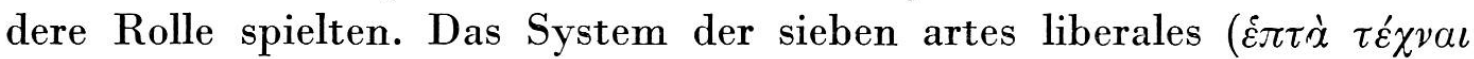

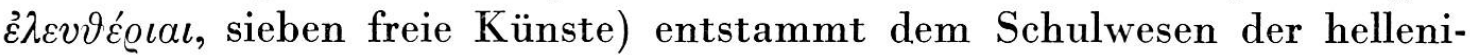
stischen Zeit und findet sich schon im ersten vorchristlichen Jahrhundert bei Dionysios Thrax, der als Verfasser der ersten griechischen Grammatik bekannt ist. Man versteht seither unter den artes liberales die Gesamtheit folgender Wissenschaften: Grammatik, Rhetorik, Dialektik, Arithmetik, Geometrie, Astronomie und Musik. Diese sieben Fächer bildeten den stofflichen Inhalt des mittleren Schulwesens in der späteren Antike. Ein Elementarunterricht ging natürlich voraus, den Abschluß der Studien bildete der Unterricht in Medizin, Rechtsgelehrsamkeit oder Philosophie. Die Ge-

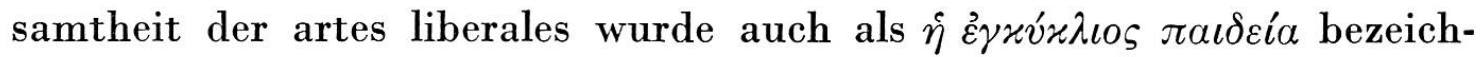
net, was verschieden übersetzt werden kann: es bedeutet entweder einen

8 Topik, Z6, 145 a.

9 Politikos, St. 258 C.

10 Friedrich Ueberweg, Grundriß der Geschichte der Philosophie, Teil 1, herausgegeben von Karl Praechter, 12. Auflage, 1926 (= Nachdruck 1957) S. 332.

11 Ebenda, S. 71.

12 Politeia, St. $530 \mathrm{ff}$.

13 John Edward Wise, The Nature of the Liberal Arts, Milwaukee 1947. - Artes liberales. Von der antiken Bildung zur Wissenschaft des Mittelalters. Herausgegeben von J. КосH, Leiden 1959 (= Studien und Texte zur Geistesgeschichte des Mittelalters, 5). 


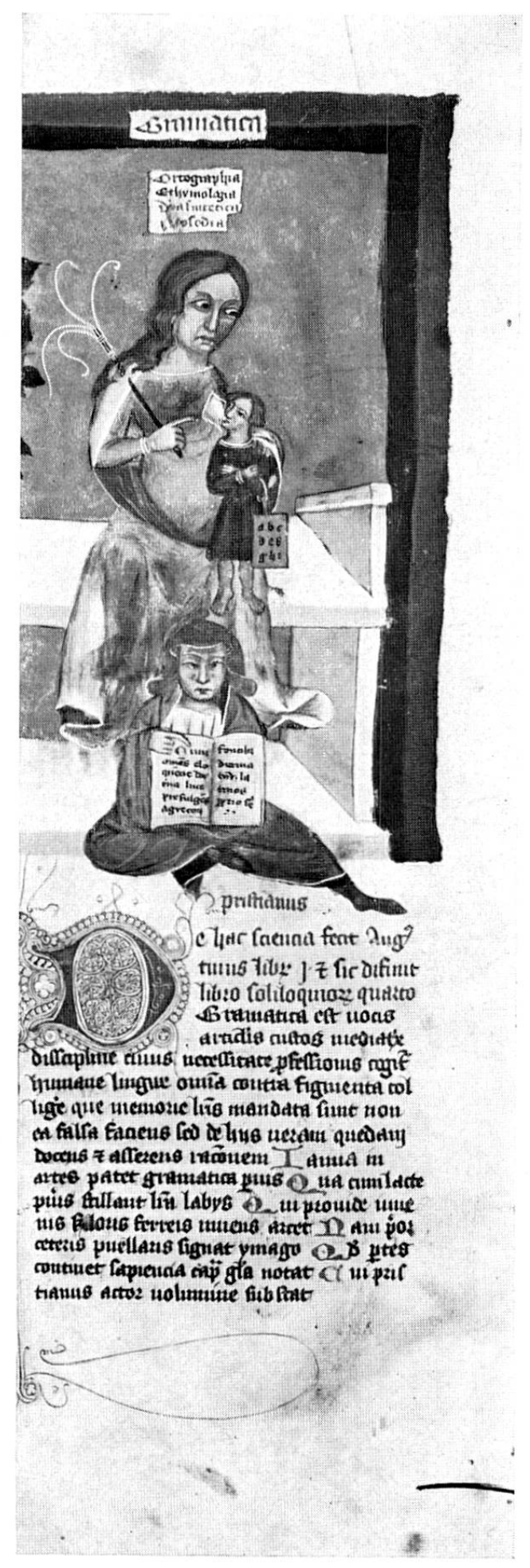

Abb. 3. Allegorische Darstellung der Grammatik

(Österreichische Nationalbibliothek, Cod. S. n. 2639, fol. 34v) 


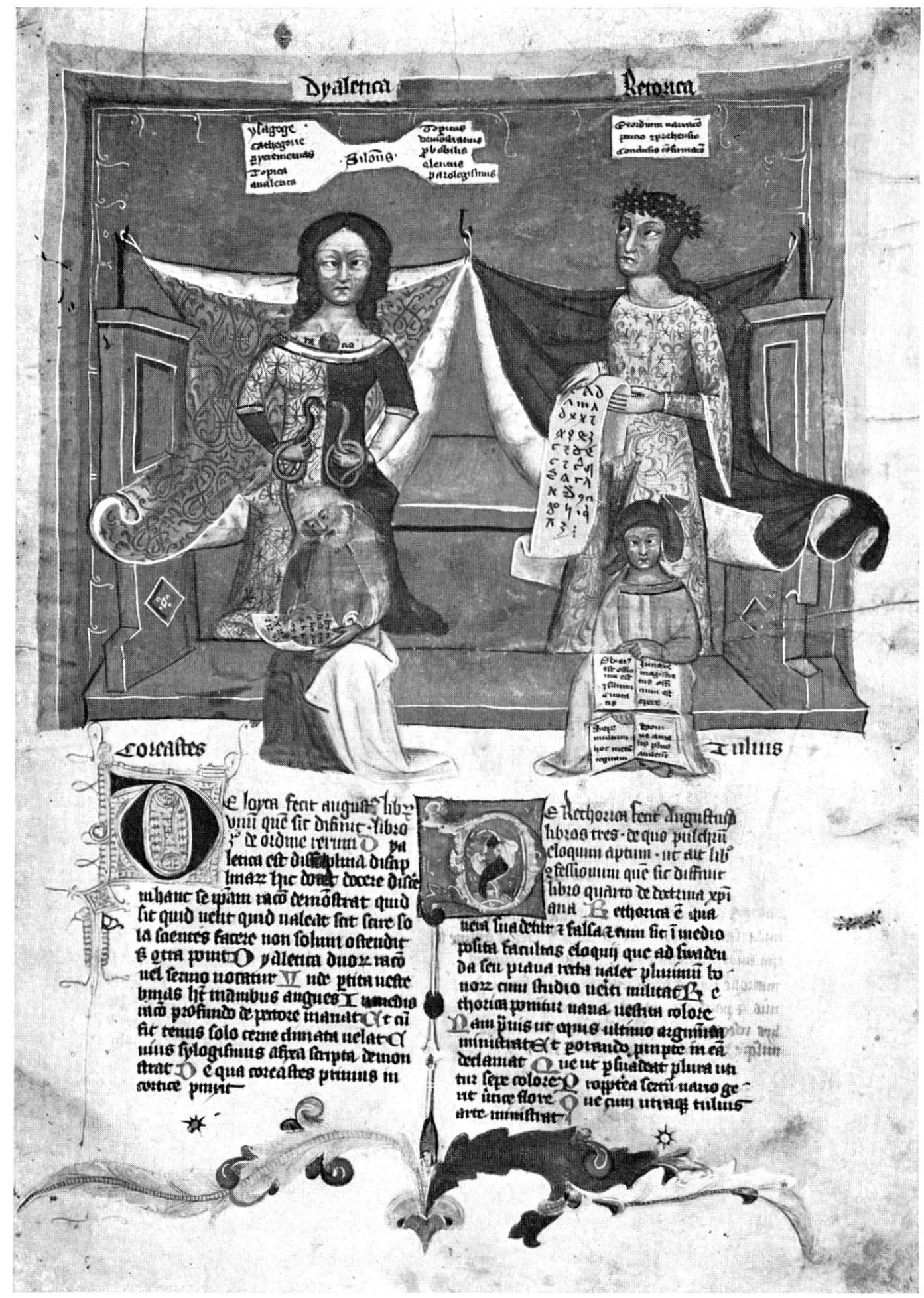

Abb. 4. Allegorische Darstellung der Dialektik (links) und der Rhetorik (rechts) (Österreichische Nationalbibliothek, Cod. S. n. 2639, fol. 35r) 


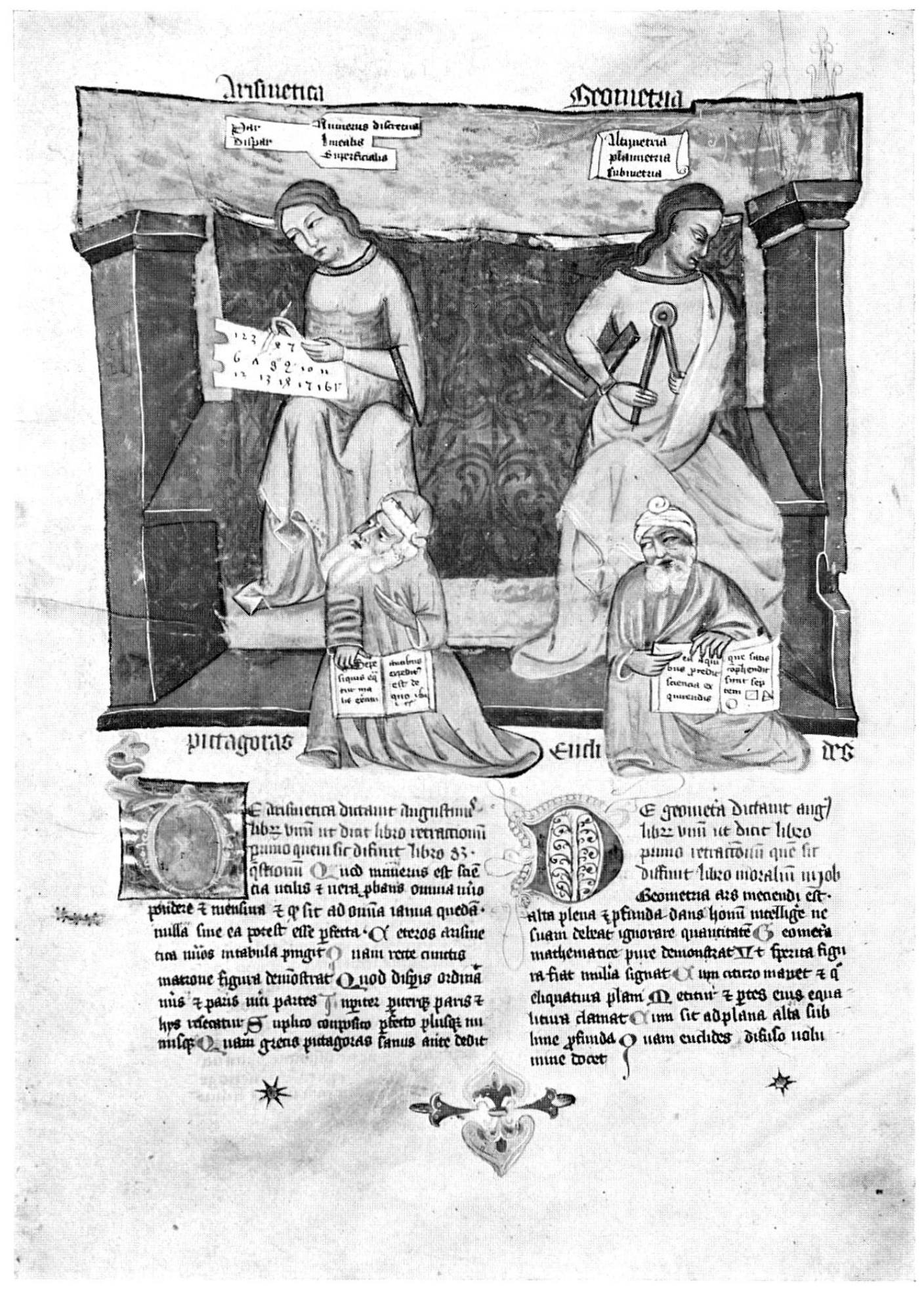

Abb. 5. Allegorische Darstellung der Arithmetik (links) und der Geometrie (rechts)

(Österreichische Nationalbibliothek, Cod. S. n. 2639, fol. 35v) 


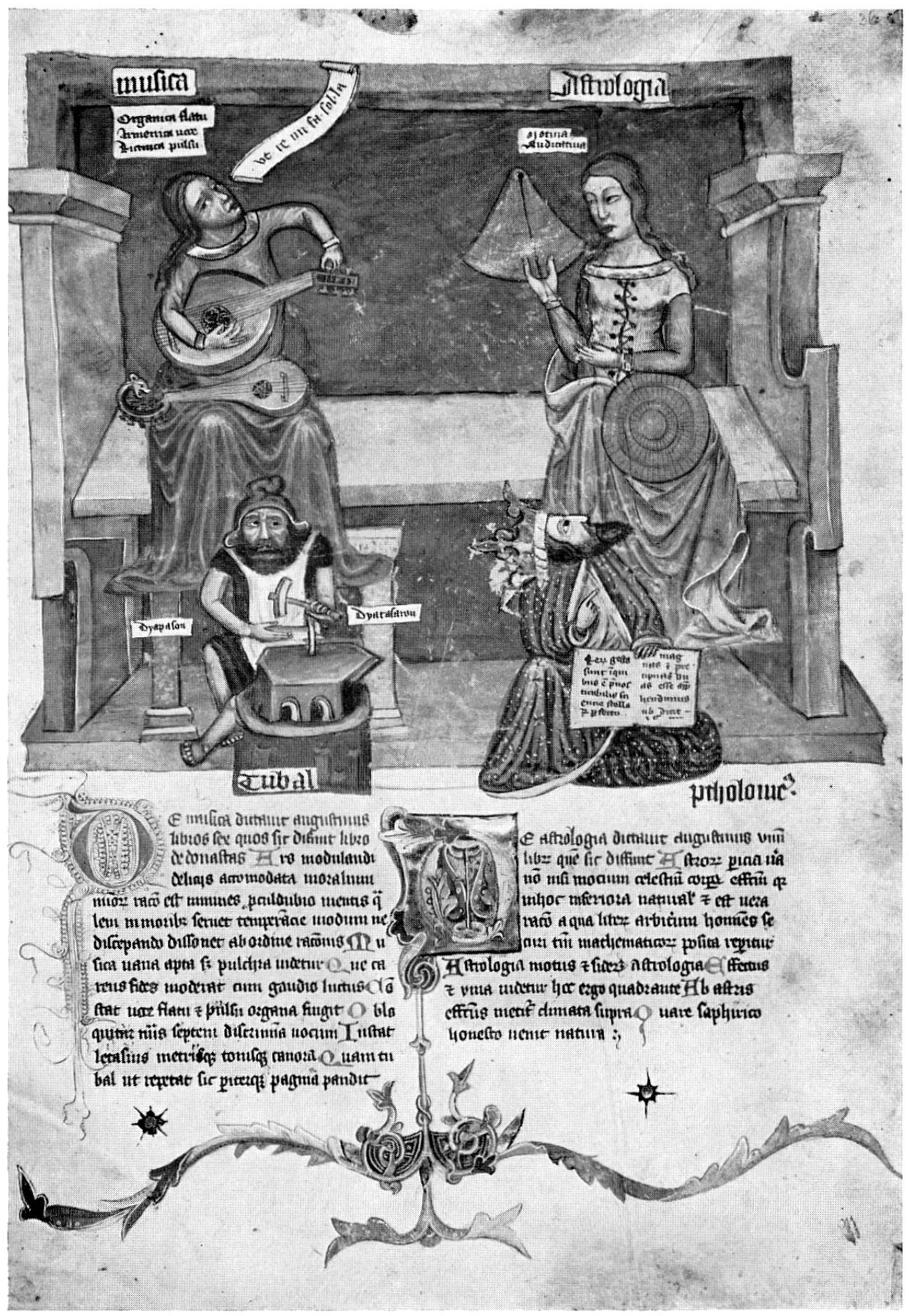

$A b b$. 6. Allegorische Darstellung der Musik (links) und der Astrologie (rechts) (Österreichische Nationalbibliothek, Cod. S. n. 2639, fol. 36r) 
Kreis von Disziplinen, die enger zusammengehören, oder die allgemein übliche, landläufige Erziehung. Daraus entwickelte sich das Wort Enzyklopädie. Jedenfalls waren die artes liberales in der römischen Kaiserzeit die Grundlage jeglicher Bildung und fanden entsprechende Berücksichtigung bei Schriftstellern wie Varro, Seneca und später bei Boethius, Cassiodor, Isidor von Sevilla. Martinus Capella verfaßte eine allegorische Verserzählung Die Hochzeit des Mercurius und der Philologie mit einer breiten Darstellung der artes liberales.

Aus einer den praktischen Bedürfnissen des mittleren Unterrichtes der hellenistischen Zeit entstammenden Aufzählung von Unterrichtsgegenständen war damit ein Kanon der Wissenschaften geworden, der sich durch mehr als tausend Jahre unverändert halten konnte, obwohl sein sachlicher Wert dem Ansehen, das er genoß, kaum entsprach. Um so interessanter ist es, diese Verhältnisse vom Standpunkt der Geistesgeschichte näher zu untersuchen und insbesondere die Gründe für die fast unlösbar erscheinende Verbindung dieser sieben Disziplinen zu beleuchten. Zunächst seien aber allegorische Darstellungen der sieben artes liberales aus einer mittelalterlichen Handschrift gezeigt. Sie finden sich im Anhang zu einem Huldigungsgedicht für König Robert von Neaped, geschrieben um 1340; der Verfasser ist wahrscheinlich Convenevole da Prato, der Lehrer Petrarcas (Abb. 3 bis 6$)^{14}$.

Nun einige Überlegungen zu den artes liberales.

1. artes. Die angegebenen Disziplinen sind also keine Philosophie, keine

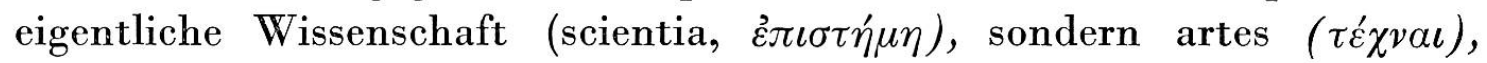
also Fertigkeiten. Das ist so zu verstehen, daß sie ursprünglich nur als Mittelstufe des allgemeinen Bildungsganges aufgefaßt wurden, weil sie die Voraussetzungen für die höheren Studien bildeten. Im frühen Mittelalter verfielen diese aber, die Akademie in Athen wurde geschlossen, das Bild eines Studienganges ging verloren, die artes allein blieben übrig und wurden als das höchste Bildungsziel angesehen.

2. artes liberales. Die eines freien Mannes würdigen Fertigkeiten, zum Unterschied von den unfreien Wissenschaften, den «artes illiberales», für die es bezeichnenderweise kein feststehendes System gab. Man rechnete dazu etwa die Webkunst, Waffenschmiedekunst, Schiffahrt, Landwirtschaft, Jagd, Heilkunst und sogar die Schauspielkunst.

3. In sachlicher Hinsicht muß bei der Betrachtung der sieben artes libe-

14 Österreichische Nationalbibliothek, Cod. Series nova 2639, fol. 34v bis $36 \mathrm{r}$. 
rales auffallen, daß es sich nur um ein rudimentäres System der Wissenschaften handelt, fehlen doch etwa Geschichte, Geographie, die eigentliche Physik, Botanik, Zoologie, Mineralogie und natürlich auch die Chemie. Daß die Musik nach heutiger Auffassung überhaupt nicht in diese Aufzählung paßt, sei ebenfalls vermerkt. Interessant ist, daß die Rhetorik noch immer aufscheint, obwohl sie jedenfalls im frühen Mittelalter viel weniger Bedeutung hatte als in der juristischen Praxis der Antike. Sie wandelte sich von einer «ars eloquentiae», also der eigentlichen Beredsamkeit, zur «ars dictaminis» für den Kanzleigebrauch.

4. Trivium und Quadrivium. Die sieben artes liberales werden üblicherweise in zwei Gruppen eingeteilt. Die ersten drei Disziplinen (Grammatik, Rhetorik, Dialektik) sind sprachlich orientiert, die übrigen vier neigen der Mathematik zu (Arithmetik, Geometrie, Astronomie, Musik). Cassiodor bezeichnete im 5 . Jahrhundert die erste Gruppe als eigentliche artes oder «scientiae sermocinales», die zweite als «disciplinae» oder «scientiae reales». Darin liegt natürlich die Wurzel für die Unterscheidung von Geisteswissenschaften und Naturwissenschaften. Interessant ist, daß CassioDOR das Wort scientiae als übergeordneten Begriff benützt und die Bezeichnung artes, d.h. Fertigkeiten, nur für die sprachlichen Unterrichtsgegenstände verwendet, was einer gewissen inneren Berechtigung nicht entbehrt. Diese Bezeichnung setzte sich jedoch nicht durch. Die Begriffe artes und scientiae, Künste und Wissenschaften, wurden bis in die Neuzeit in übergreifender Bedeutung verwendet und haben erst im 19. Jahrhundert die heute übliche Abgrenzung erfahren.

Wenn sich auch die Bezeichnung Cassiodors nicht durchgesetzt hat, so doch die von ihm verwendete Einteilung. In der einfachen, jedoch plastischen Sprache des frühen Mittelalters bürgerte sich für die Gruppe Grammatik, Rhetorik, Dialektik die Bezeichnung «Trivium» heraus, während Arithmetik, Geometrie, Astronomie und Musik zusammenfassend als "Quadrivium» bezeichnet wurden. Die wörtliche Bedeutung ist einerseits «Dreiweg», anderseits "Vierweg». Diese Bezeichnungen suchen also gar nicht die Gruppen sachlich zu erfassen, sondern halten sich an die Anzahl der darin vorkommenden Lehrgegenstände.

5. Zahlenspekulationen. Die Bezeichnungen Trivium und Quadrivium weisen auf einen Umstand hin, der bei der Erörterung der artes liberales bisher noch viel zu wenig beachtet worden ist. Die Beliebtheit der Artes im Mittelalter hing letzten Endes nicht nur von ihrer theoretischen oder praktischen Brauchbarkeit ab (die, wie wir gesehen haben, ernsteren Ein- 
wänden nicht standhielt), sondern viel mehr vom Mitschwingen gewisser Zahlenspekulationen, wie sie bekanntlich seit der Spätantike unter dem Einfluß des Neuplatonismus und der Neupythagoreer große Bedeutung erlangten ${ }^{15}$. Danach war z.B. die Zahl 1 ein Symbol für Gott, die Zahl 3 bezog sich auf die Seele (die in Vernunft, Verstand und Mut eingeteilt wurde), die Zahl 4 bezog sich auf die Welt (es gibt 4. Weltrichtungen, 4 Jahreszeiten, 4 Elemente usw.). Auch die Zahl 7 nahm eine ausgezeichnete Rolle ein (es gab 7 Planeten, 7 Weltwunder, 7 Städte, die sich darum stritten, Geburtsort Homers zu sein, usw.), die durch das Christentum noch verstärkt wurde, da ja entsprechend der Weltschöpfung 7 Wochentage eingeführt wurden usw. ${ }^{16}$. Erhöht wurde die Dignität der 7 dadurch, daß sie sich als Summe von $3+4$ ergibt. Nach der Interpretation von AuguStivus bedeutet dies die Summe eines geistigen (3) und eines weltlichen Prinzips (4) als Totalität der Schöpfung ${ }^{17}$. Eine analoge Zerlegungsmöglichkeit der 7 findet sich in zahlreichen Beispielen, angefangen von den 7 Bitten des Vaterunsers bis zu den 7 Kurfürsten, unter denen bekanntlich 3 geistliche und 4 weltliche waren. Was war naheliegender, als daß die Zahl der artes liberales gerade 7 betragen mußte und daß sie in die Gruppen des Trivium und Quadrivium zerfielen? Die Zusammenfassung der sprachlich orientierten und der mathematisch orientierten Wissenschaften ergab deren Gesamtheit, so daß das Gesamtwissen eine wunderbare Harmonie darstellte. So brachten schon Cassiodor und später Dante (im Convivio) die artes liberales in direkte Beziehung zu den Planeten, und zwar das Trivium zu den inneren, das Quadrivium zu den äußeren Planeten. Ähnlich wie gewisse Werbeslogans der heutigen Zeit außer ihrer sachlichen Aussage eine zusätzliche, rational nicht ohne weiteres erklärbare Wirkung besitzen, so hatten auch die 7 artes liberales, eben weil es ihrer 7 waren, einen ungemein kompakten Zusammenhang und wurden noch lange verwendet, als sachlich nicht mehr der geringste Anlaß dazu vorlag.

15 Vincent Foster Hopper, Mediaeval Number Symbolism. Its Sources, Meaning, and Influence on Thought and Expression, New York 1938 (= Columbia University Studies in English and Comparative Literature, 132). - Erich Schöner, Das Viererschema in der antiken Humoralpathologie, Wiesbaden 1964 (= Sudhoffs Arch. Gesch. Med. Naturwiss., Beiheft 4). - SEYYed Hossein NASR, An Introduction to Islamic Cosmological Doctrines, Cambridge (Mass.) 1964.

16 Herman Polzer-van Kol, Priester Arnolds Gedicht von der Siebenzahl (Ausgabe, Schreibung, Reime) Bern 1913 (= Sprache und Dichtung. Forschungen zur Linguistik und Literaturwissenschaft 13).

17 Vgl. Hopper l.c., S. 84; Augustinus, Civitas Dei XX, 5. 
Wem der Bezug eines Systems der Wissenschaften auf neupythagoreische Zahlenspekulationen nicht ohne weiteres einleuchtet, den möchte ich auf ein Beispiel aus unserer Zeit verweisen. Noch heute wird in den angelsächsischen Ländern das Ziel des Elementarunterrichtes in populärer Weise als die Beherrschung der 3 «r» hingestellt, worunter verstanden ist: reading, writing, 'rithmetic. Die mnemotechnisch leichter zu beherrschende Dreiheit der unkorrekt ausgesprochenen Wörter setzt sich über die Rechtschreibung glatt hinweg.

6. Die Artistenfakultät. Als seit dem 11.Jahrhundert die arabische Wissenschaft in Europa eindrang und das Interesse an den antiken Quellen erwachte, entstanden die Universitäten, die zu einem wichtigen Faktor bei der Beibehaltung der artes liberales wurden. Diese wurden nämlich an der Facultas artium liberalium unterrichtet, kurz «Artistenfakultät» genannt. Ihr Besuch bot die Voraussetzung für das Studium an den übrigen Fakultäten für Jurisprudenz, Medizin oder Theologie. Die Artistenfakultät hat viele Jahrhunderte existiert und ist erst später durch die philosophische Fakultät abgelöst worden.

Wir haben nun die Voraussetzungen für ein Verständnis von Geßners System der Wissenschaften ${ }^{18}$ (Abb.7). Die Philosophie umfaßt als Inbegriff der Künste und Wissenschaften zwei Hauptgruppen: die praeparantes und die substantiales, also die vorbereitenden und die Hauptwissenschaften. Erstere zerfallen in die necessaria und die ornantes, also in die notwendigen und die «schmückenden», d.h. zusätzlichen Wissenschaften. Für die necessaria übernimmt Geßner die Einteilung Cassiodors in die sermocinales (also im wesentlichen das sprachlich orientierte Trivium) und die mathematischen Wissenschaften (das Quadrivium), fügt aber in beiden Gruppen noch ein Wissensgebiet hinzu: Unter den mathematischen Wissenschaften gibt es nun außer der Astronomie auch die Astrologie - ein sehr bedeutsamer Hinweis für die beginnende Trennung der beiden Wissensgebiete; daß sie aber noch lange nicht vollständig war, geht schon daraus hervor, daß viele Jahrzehnte später KePLER noch Horoskope gestellt hat. Auch das frühere Trivium ist um ein Gebiet erweitert: die Poesie. Allerdings ist Geßner im Zweifel, ob er sie zu den sermocinales rechnen soll; die Poesie ist zwar sprachlich orientiert, zählt aber nicht zu den Notwendigkeiten und

18 Partitiones theologicae, S. (16). Zum Vergleich diene etwa das lückenhafte System der Wissenschaften bei JoAchim Fortius Ringelberg, Lucubrationes, vel potius absolutis-

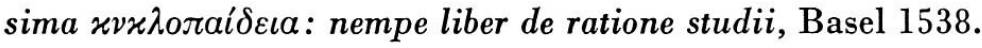




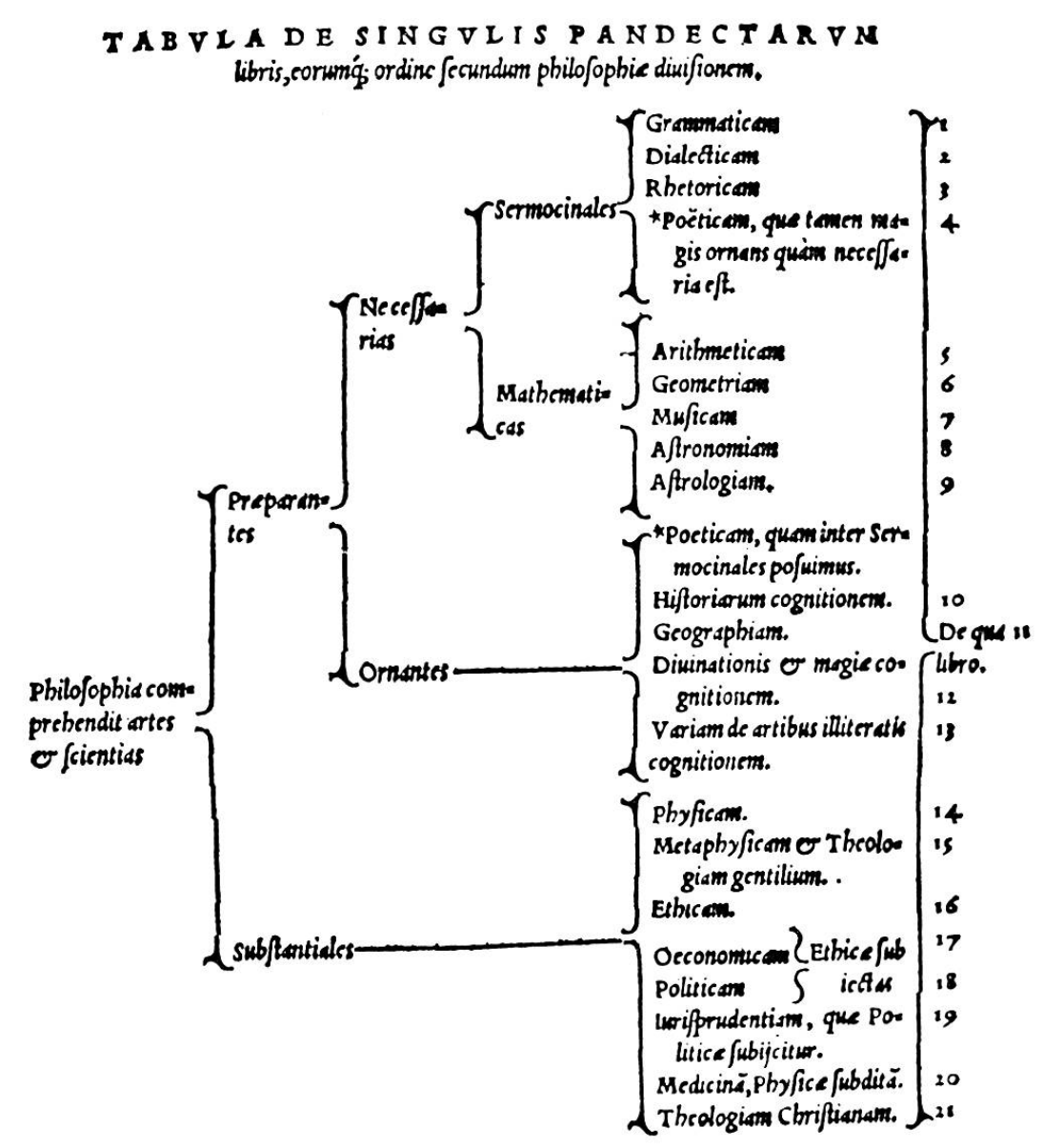

$A b b .7$. Geßners System der Wissenschaften in den «Pandekten»

hätte daher eher bei den ornantes Platz, weshalb sie auch dort aufgeführt ist. $\mathrm{Da} \beta$ die Poesie überhaupt zu den lehrbaren Wissensgebieten gerechnet wurde, darf nicht befremden; es entsprach durchaus dem Geist der damaligen Zeit, wenn etwa Conrad Celtes Professor für Mathematik und Poesie in Wien war.

Jedenfalls hatte Geßner keine Hemmungen, die Siebenzahl der artes aufzugeben und ihr System, das zu einer Fessel geworden war, zu sprengen. Das ist um so bemerkenswerter, als die Zahlensymbolik zu seiner Zeit noch durchaus verbreitet war und sich noch ein halbes Jahrhundert später, z.B. bei dem Dichter Edmund Spenser, findet ${ }^{19}$. Geßner erhielt nun

19 A.Kent HieatT, Short Time's Endless Monument: The Symbolism of Numbers in Edmund Spenser's «Epithalamion», New York 1960. - Alastair Fowler, Spenser and the Numbers of Time, London 1964. 
die bunt zusammengewürfelte Gruppe der ornantes, umfassend: Poesie (mit Vorbehalt), Geschichte, Geographie, Weissagung und Magie, sowie die artes illiberales, also Technik im weitesten Sinn. Es handelt sich naturgemäß um jene Gebiete, die im Zeitalter der Renaissance eifrig betrieben wurden.

Die Gruppe der substantiales enthält wohl die drei traditionellen Fächer Jurisprudenz, Medizin und Theologie, aber mit wesentlichen Änderungen: Hauptfächer sind nunmehr Physik, Metaphysik mit Einschluß der heidnischen Theologie, Ethik, christliche Theologie. Die Medizin ist der Physik untergeordnet, die Jurisprudenz der «Politik» und diese sowie die Ökonomie der Ethik.

Geßner hat also in seinen «Pandekten» versucht, das System der artes liberales, das den sich stürmisch erweiternden Kenntnissen seiner Zeit nicht mehr entsprach, durch verschiedene Erweiterungen und Anpassungen irgendwie zu retten, ohne die traditionellen Grundlagen ganz zu verlassen. Im Laufe der späteren Jahrhunderte wurden natürlich noch weitere Systeme der Wissenschaften aufgestellt, die von ganz anderen Voraussetzungen ausgingen, z.B. von Francis Bacon, Auguste Comte oder die Dezimalklassifikation von Melvil Dewey. Aber noch 250 Jahre nach Geßner teilte J.J.Eschensurg ${ }^{20}$ die Wissenschaften folgendermaßen ein: 1. philologische, 2. historische, 3 . philosophische, 4 . mathematische, 5. physische, 6. medizinische, 7. juristische, 8. theologische. Wenn man diese Aufstellung betrachtet, kann man auch hier wieder das alte System der artes liberales hindurchschimmern sehen: 1 bis 3 entspricht dem Trivium, nur daß statt der Rhetorik die Geschichte aufgenommen ist und die Dialektik zur Philosophie erweitert wurde; es ist die Gruppe der philosophisch-historischen Wissenschaften. 4 bis 5 entspricht dem Quadrivium, wobei natürlich die Musik fehlt. Zu den mathematischen Wissenschaften rechnet Eschenburg auch die bürgerliche Baukunst und die Kriegswissenschaften, zu den physischen Wissenschaften Zoologie, Botanik und Mineralogie, die, wie üblich, als Naturhistorie zusammengefaßt werden. 4 und 5 bilden also die mathematisch-naturwissenschaftliche Gruppe. Die drei letzten Fächer entsprechen dann den bekannten Universitätsfakultäten. Eschenburg hält sich also noch mehr als Geßner an die Tradition.

Untersuchungen über die verschiedenen Systeme der Wissenschaften, wie wir sie eben durchgeführt haben, wurden vor nicht allzulanger Zeit als

20 Johann Joachim Eschenburg, Lehrbuch der Wissenschaftskunde, Berlin 1792. 
ein eigener Wissenschaftszweig, genannt «Enzyklopädie», angesehen. In jedem älteren Konversationslexikon kann man dieses Stichwort finden, doch ist heute die ursprüngliche Bedeutung dieses Wortes dem Bewußtsein der meisten Gebildeten entschwunden. Noch im 19. Jahrhundert gab es eifrige Diskussionen darüber, aber das Interesse an ihnen ist jetzt vielfach erlahmt. Die Ursache für diese sehr bezeichnende Tatsache liegt wohl darin, daß die Spezialisierung der Forscher so weit fortgeschritten ist, daß nur mehr wenige einen Ủberblick über das Gesamtgebiet der Wissenschaften besitzen. Daß aber enzyklopädische Untersuchungen einen unmittelbaren Zusammenhang mit geistigen Problemen unserer Zeit haben, sei an zwei Beispielen aufgezeigt:

1. Nach obigen Ausführungen wird man vielleicht meinen, daß der Begriff der artes liberales nur eine historische Bedeutung besitzt; dem ist aber nicht so. In den angelsächsischen Ländern ist der Begriff «liberal arts » als einer mittleren Stufe der allgemeinen Bildung heute noch sehr lebendig, er wird vielfach mit «Geisteswissenschaften» identifiziert. Dieses Weiterleben der artes liberales hängt damit zusammen, daß viele Begriffsbildungen, die vom deutschen Idealismus und vom französischen Positivismus geprägt wurden, in das europäische Erziehungs- und Bildungswesen eingedrungen sind, jedoch nicht in das anglo-amerikanische. In der Geschichte der Philosophie ist diese Tatsache schon festgestellt worden. Weniger bekannt ist, daß heute noch eine weitgehende Diskrepanz der philosophischen und naturwissenschaftlichen Nomenklatur besteht, die es bis zum 18. Jahrhundert nicht gegeben hatte. So haben Ausdrücke wie Wissenschaft, Naturwissenschaften, Geisteswissenschaften, ja selbst Technik kein exaktes Äquivalent im Englischen. Umgekehrt sind Ausdrücke folgender Art schwer zu übersetzen : liberal arts college, bachelor of arts, liberal arts education, Museum of Arts and Industries, natural philosophy (nicht Naturphilosophie, sondern theoretische Physik), philosophical instruments (physikalische Apparate) usw. Das Englische hat die bis in die ausgehende Barockzeit verwendete einheitliche lateinische Terminologie (darunter auch artes liberales) länger beibehalten als das Deutsche oder Französische.

2. Nicht aus bibliographischen Gründen, sondern aus der Notwendigkeit, das Universitätsstudium den heutigen Bedürfnissen anzupassen, steht die Universitätsverfassung und damit die Einteilung der Wissenschaften wieder zur Diskussion, besonders im Zusammenhang mit der Errichtung neuer Universitäten. Die artes liberales sind natürlich im einzelnen überholt, aber in der philosophischen Fakultät leben sie weiter. Im Gegensatz zur Termino- 
logie sind in Europa die herkömmlichen institutionellen Verhältnisse im allgemeinen beibehalten worden (abgesehen etwa von der Abspaltung medizinischer Akademien oder der mathematisch-naturwissenschaftlichen Fakultät), während in den angelsächsischen Ländern gerade hier neue Wege beschritten wurden.

Die Beschäftigung mit dem enzyklopädischen System Geßners führt somit direkt in vieldiskutierte Probleme unseres Zeitalters. Es ist dies wohl ein Beweis für die geistige Bedeutung eines Mannes, dessen Todestag sich nun zum 400. Mal jährt, anderseits aber auch ein Zeichen für die innige kulturelle Verbundenheit des Abendlandes über Jahrhunderte hinweg. 